\title{
The estimation of genetic parameters for growth curve traits in Raeini Cashmere goat described by Gompertz model
}

\author{
Heydar Ghiasi ${ }^{\mathrm{a}, *}$, T.M. Lupi ${ }^{\mathrm{b}}$, M.S. Mokhtari ${ }^{\mathrm{c}}$ \\ ${ }^{a}$ Department of Animal Science, Faculty of Agricultural Science, Payame Noor University, Tehran, Iran \\ b Escola Superior Agraria do Instituto Politecnico de Castelo Branco, 6001-909, Castelo Branco, Portugal \\ ${ }^{\mathrm{c}}$ Department of Animal Science, Faculty of Agriculture, University of Jiroft, P.O. Box 364, Jiroft, Iran
}

A R T I C L E I N F O

\section{Keywords:}

Body weight

Age

Non-linear function

Animal model

Genetic evaluation

\begin{abstract}
A B S T R A C T
The objectives of this study were to describe growth curve of Raeini Cashmere goat applying the Gompertz growth model and genetic evaluation of growth curve-related traits including model parameters of A, B and K, inflection age (IA) and inflection weight (IW) under animal model. The data used in this study, collected in Raeini Cashmere goat breeding station from 1997 to 2009 and were included 12,831 body weights records measured at birth, weaning, 6-months of age, 9-month of age and yearling of age. The Pearson's correlation coefficient between observed and predicted body weights was 0.98 , which means that Gompertz model adequately described the growth curve in Raeini Cashmere goat. The estimated value for growth curve parameters of A, B and K were 17.97, 1.97 and 0.017, respectively. The weight and age at point of inflection were $6.63 \mathrm{~kg}$ and 52.94 days, respectively. Direct heritability estimates for A, B, K, IA and IW were low values of $0.14,0.10$, $0.03,0.14$ and 0.14 , respectively. Low estimated values for direct heritability of the studied growth curve traits in Raeini Cashmere goat indicated that direct selection for these traits may not be useful in terms of achieving genetic change. Direct genetic correlations ranged from -0.76 (K-IW) to 0.98 (A-IW). Phenotypic correlation estimates were generally lower than the direct genetic ones and ranged from -0.30 (K-IW) to 0.69 (A-B and BIA). IA and IW had high positive phenotypic (0.86) and genetic (0.99) correlations, implying IA and IW were highly correlated in terms of phenotypic and genetic effects. The studied growth curve parameters of Raeini Cashmere goat have shown low levels of additive genetic variation.
\end{abstract}

\section{Introduction}

Raeini Cashmere breed is one of the most important Iranian native breeds of goat which have been raised in Kerman province, southeastern part of Iran, by nomadic flock holders. High valuable Cashmere produced by this breed contributes considerably to agricultural economy of flock holders in this region of Iran. The relationship between body weight and the age of animal can be modeled by growth curves (Keskin et al., 2010). Fitting growth curve provides some parameters that explain the pattern of animal growth over its life. Growth curve parameters are useful for estimating several important characteristics such as animal weight at a certain age, optimum slaughter age of animal, mature body weight, growth rate and also assessing the management factors affecting growth such as feed requirements (Daskiran et al., 2010).

As animal grows its growth rate changes, which is reflected in the growth curve curvature, until it reaches the highest growth rate point. After this inflection point, the growth rate gradually decreases. This tendency continues until growth is stabilized and it reaches its maximum value that coincides with the horizontal asymptote (Fitzhugh, 1976; Gomez et al., 2008; Lupi et al., 2016). The growth curve can help for planning farm management strategies and decision making on the culling of poor producers and selecting the highly productive animals only by taking their growth curve into account (Waheed et al., 2011). Several studies showed that growth curve parameters are highly heritable and can be used in developing genetic selection strategies (Mignon-Grasteau et al., 2000; Lupi et al., 2016). Different non-linear mathematical functions such as negative exponential (Brown et al., 1976), Brody (Brody, 1945), Gompertz (Laird, 1965), Logistic (Nelder, 1961), Bertalanffy (Bertalanffy, 1957) and Richards (Richards, 1959) have been used to describe the growth curve.

Waheed et al. (2011) estimated growth curve parameters in Beetal goats applying Brody and Gompertz models. They concluded that any of these models can be used for estimating growth curves of Beetal breed. Several studies reported that Gompertz model is an appropriate model for fitting growth curve in goat (Sghaier et al., 2007; Kume and Hajno,

\footnotetext{
* Corresponding author.

E-mail address: ghiasi@ut.ac.ir (H. Ghiasi).
} 
2011; Gaddour et al., 2012).

To our knowledge there is no previous investigation regarding the studying of growth curve in Raeini Cashmere goat breed. Therefore, the objective of the present research was to study the growth curve in Raeini Cashmere goat breed applying Gompertz model. Furthermore, growth curve-related traits were evaluated genetically; direct heritability, genetic and phenotypic correlations among them were estimated applying animal models.

\section{Material and methods}

\subsection{Data}

A total of 12,831 body weight records that collected from 1997 to 2009 in Raeini Cashmere goat breeding station, located in Baft city, Kerman province, south-eastern part of Iran, were used in the present study. Records included birth-weight, weaning-weight, 6-month body weight, 9-month body weight and yearling weight. The weaning age of kids was 80 to 95 days old. Animals that had less than four records for body weights, animals without any records and body weights outside of the range mean $\pm 2 \times$ S.D. have been removed from the data set. After editing the primary data set, a total of 4746 body weight records belong to 1000 animals were obtained and used for estimating the parameters of growth curve. Distributions of the numbers of animals across the considered fixed effects, except for birth year, are shown in Table 1. The lowest and highest numbers of animals were obtained in birth year of 2009 (33 goats) and birth year of 2005 (240 goats), respectively.

\subsection{Statistical models}

The non-linear Gompertz growth curve model was fitted on body weight-age data applying NLIN procedure and the Newton-Gauss method (Statistical Analysis System (SAS, 2004) as follows:

$w(t)=A \times \exp (-B \times \exp (-K \times t))$

Where, $w(t)$ is the observed body weight at age $t$ (in $\mathrm{kg}$ ), $\mathrm{t}$ is age in days, A, B and $\mathrm{K}$ are model parameters. The parameter A defines as asymptotic value or predicted mature live weight; the parameter B is the turning point of growth and parameter $\mathrm{K}$ represents the growth rate. This model was fitted for each animal and then animals that did not converge or have the model parameter of $\mathrm{A}>30$ were removed from the data set. The inflection weight (IW) and inflection age (IA) were calculated as follows (Lupi et al., 2016):

$\mathrm{IW}=\mathrm{A} / 2.72$

and

$\mathrm{IA}=\ln (\mathrm{B}) / \mathrm{K}$

The estimation of (co)variance components for the studied growth curve traits was carried out by fitting the below animal model on growth curve traits of 1000 kids applying WOMBAT software (Meyer, 2007):

$\mathbf{y}=\mathbf{X b}+\mathbf{Z}_{1} \mathbf{a}+\mathbf{e}$

Where, $\mathbf{y}$ is a vector of records for the studied growth curve traits; $\mathbf{b}$, a and $\mathbf{e}$ are vectors of fixed, direct genetic and residual effects, respectively. Common fixed effects included in the animal models for the studied growth curve traits were sex of kids in 2 classes (male and female), dam age at lambing in 6 classes (2-7 years old), birth type in 2 classes (single and twin) and birth year in 13 classes (1997-2009). The comparison of means and least squares means for the studied traits across the levels of fixed effects were carried out by GLM procedure of SAS 9.1 software (Statistical Analysis System (SAS, 2004). Genetic, phenotypic and residual correlations between the studied traits were estimated under bivariate animal models.

\section{Result and discussion}

\subsection{General considerations}

Least squares means for the studied growth curve traits of Raeini Cashmere goat across the considered fixed effects are presented in Table 1. In the present study, birth year of kids had significant effect on all the studied growth curve traits $(\mathrm{p}<0.01)$. The maximum values for A $\quad(22.41 \pm 0.43), \quad$ B $(2.16 \pm 0.02), \quad \mathrm{K} \quad(0.04 \pm 0.002), \quad$ IA $(2.16 \pm 0.02)$ and IW $(8.27 \pm 0.016)$ were obtained in birth year of 1999. The minimum values for A (14.01 \pm 0.35$)$, IA $(26.28 \pm 2.50)$ and IW (5.17 \pm 0.13$)$ were obtained in birth year of 1998 while minimum ones for $\mathrm{Bp}(1.71 \pm 0.02)$ and $\mathrm{K}(0.011 \pm 0.002)$ were obtained in birth years of 2005 and 2004, respectively. The differences between maximum and minimum values for the studied growth curve traits of Raeini Cashmere goat were statistically significant ( $p<0.01)$.

Table 1

Least squares means ( \pm S.E.) for the studied growth curve traitsof Raeini Cashmere goat.

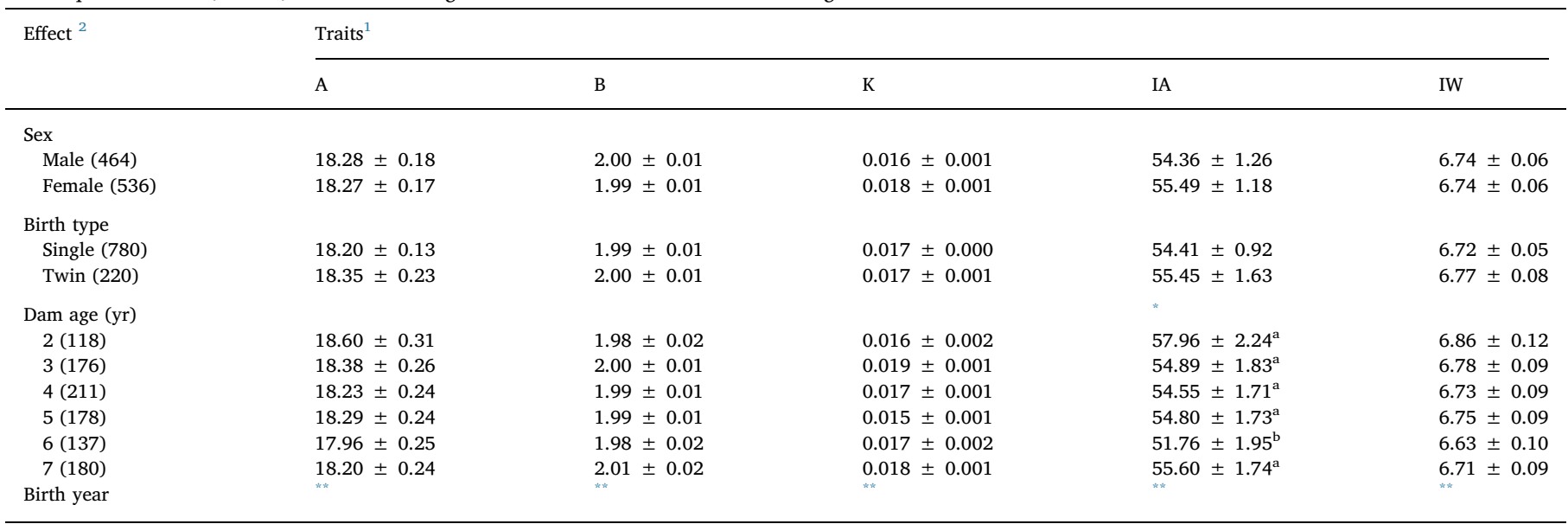

Means with similar letters in each sub class within a column do not differ from another at $\mathrm{p}<0.05$.

$* \mathrm{p}<0.05$.

** $\mathrm{p}<0.01$

${ }^{1}$ A: Predicted mature live weight, B: Turning point of growth, K: Relative growth rate, IA: Inflection age, IW = Inflection weight.

${ }^{2}$ The numbers of animal per each level of fixed effect are shown in the parenthesis. 
Different climatic variations and managerial practices through the years may influences on the growth of animals. Similarly, Sghaier et al. (2007) reported significant effect of year on growth curve traits of Tunisian local kids.

Sex and birth type of kids had no significant effects on all the studied growth curve traits of Raeini Cashmere goat ( $p>0.05$ ). Similar to us, Waheed et al. (2011) reported that sex and birth type had no significant effects on the growth curve parameters of Beetal goats. Dam age had no significant effects on the considered growth curve traits ( $p>0.05$ ) except for IA $(\mathrm{p}<0.05)$. The IA of kids from 6 years old dams was significantly lower than the other age groups of dams ( $p$ 0.05). Sghaier et al. (2007) fitted Gompertz model on growth trajectory of local Tunisian kids and reported that sex, birth type of kids and dam age significantly influenced on growth curve of this breed only during two first months of age. Kume and Hajno (2011) studied growth curve variations (described by Gompertz model) of Alpine goat from birth to six-month of age and reported that sex and birth type of kids had significant effects on Parameters of A, B and K which were not in agreement with the obtained results in the present study. Such difference may be explained by breed differences, data structure and management strategy.

In the present study, Pearson's correlation coefficient between the observed values of body weights and those of predicted by Gompertz function was 0.98 , indicating that Gompertz is an appropriate function to explain the growth curve in Raeini Cashmere goat (Fig. 1). Waheed et al. (2011) and Raji et al. (2015) reported $\mathrm{R}^{2}$ values of 0.99 and 0.94 for Gompertz model in Beetal goat and Nondescript goat, respectively. In the present study, Gompertz model predicted birth weight and yearling weight accurately than weight at 3, 6 and 9-month of age. In other words, predicted body weights at 3, 6 and 9-months of age were slightly higher than those of observed. Waheed et al. (2011) concluded that Brody and Gompertz models similarly described the growth curve in Beetal goat.

\subsection{Growth curve parameters}

In the present study, after fitting the Gompertz model the obtained value of A was 17.97, which estimate the mature weight in Raeini Cashmere goat, and lower than the corresponding value reported by Waheed et al. (2011) in Beetal goat (23.39), implying that Raeini goat has a lower weight at maturity. The lower values of parameter A also estimated by Raji et al. (2015) in male (8.40) and female (6.42) Nondescript goat breed. The parameter of A in growth curve is affected by environmental and genetic effects (Narinc et al., 2010; Lupi et al., 2016). Therefore, this parameter should be compared with caution in different breeds.

The obtained value of B was 1.97, that was similar with value obtained by Waheed et al. (2011) in Beetal kids (1.98) applying Gompertz function. The parameter B is a scale parameter and have no biological interpretation. The obtained value of $\mathrm{K}$ was 0.017 , which shows the growth rate to reach the adult weight, was estimated as 0.017 in Raeini Cashmere goat. Pires et al. (2017) estimated a value of 0.0108 for parameter $\mathrm{K}$ in Repartida goat applying Gompertz model. The value obtained for parameter $\mathrm{K}$ in this study is not in agreement with Raji et al. (2015) and Waheed et al. (2011) who reported larger value of $K$ parameter under Gompertz model for Nondescript and Beetal goat, respectively. Animal with high value for parameter K attain maturity weight earlier than animals with low value for this parameters (Lupi et al., 2016). The selecting of animals for decreasing or increasing the mature weight depends on the breeding and marketing objectives. If the breeding program is designed for attaining animals with lower energy requirements, earlier maturity and lower mature weight may be desirable but if the objective of the breeding program is to satisfy the market demand with higher mature weight of animals, a later maturity should be consider for animals (Fitzhugh and Taylor, 1971). In the production system of Raeini Cashmere goat animals have been raised for meat production purposes. Therefore, animals with high mature weight and later maturity were preferred.

In the present study obtained values of IW and IA were $6.63 \mathrm{~kg}$ and 52.94 days, respectively. Najari et al. (2007) reported values of 38.15 days for age and $5.95 \mathrm{~kg}$ for weight at inflection point in local Tunisian goat. Age and weight at inflection point in Alpine goat was reported as 47 days and $9.04 \mathrm{~kg}$ (Kume and Hajno, 2011) that are larger than the obtained values in the current study. Raji et al. (2015) reported lower age and weight at inflection point $(2.18$ weeks and $3.09 \mathrm{~kg}$ ) in Nondescript goat. Brody (1945) pointed out that age at inflection point can be considered as the onset of puberty but Pittroff et al. (2008) showed that there are no relationship between onset of puberty and the inflection point of growth curve.

Age and weight at inflection point may be used to determine the optimum slaughter age. According to growth curve parameters obtained, the optimum age and weight for Raeini Cashmere goat is 52.94 days and $6.63 \mathrm{~kg}$, respectively. The most of Raeini Cashmere goat flock holders slaughter their animals at age and weight of approximately 6month old and $20 \mathrm{~kg}$, respectively (Karimkhani et al., 2005). The values estimated in this study for optimum slaughter age and weight are less than the real slaughter age and weight that is practiced in commercial Raeini Cashmere goat production system. In Iranian production system, goats are sold out according to the live body weights and not based on the carcass value (Kamjoo et al., 2014). The differences between price of animals sold out in the early and late ages is low. The obtained results in the current study showed that the application of growth curve parameters to estimate the optimum slaughter age and weight is useful when animal price system in the market to be based on the carcass value not on the live body weights value.

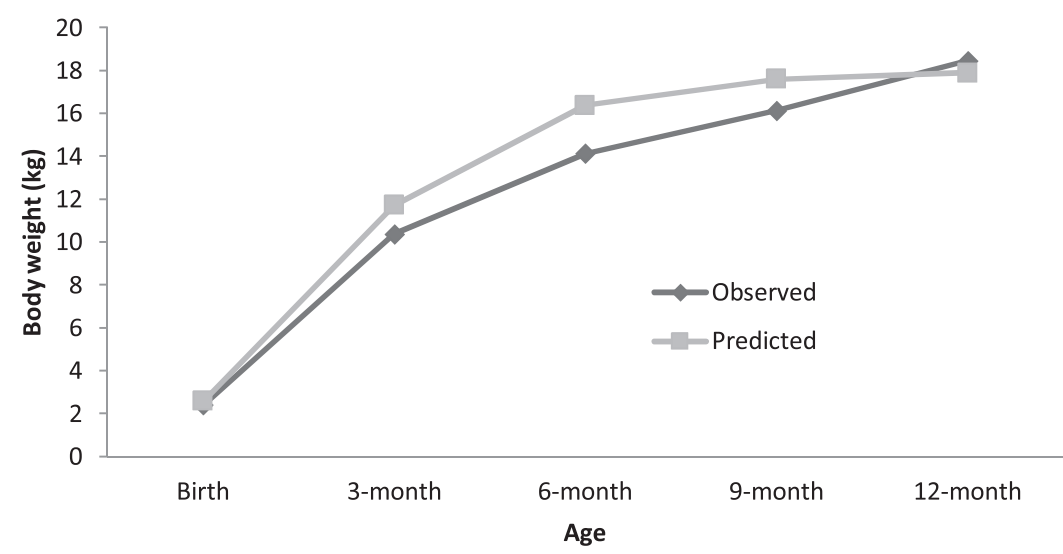

Fig. 1. Observed and predicted body weights (kg) of Raeini Cashmere goat at different ages by Gompertz model. 
Table 2

Variance components and direct heritability estimates for the studied growth curve traits in Raeini Cashmere goat.

\begin{tabular}{lllll}
\hline Traits $^{\mathrm{a}}$ & ${ }^{\mathrm{b}} \mathrm{\sigma}_{\mathrm{a}}^{2}$ & ${ }^{\mathrm{b}} \mathrm{\sigma}_{\mathrm{e}}^{2}$ & ${ }^{\mathrm{b}} \mathrm{\sigma}_{\mathrm{p}}^{2}$ & ${ }^{\mathrm{b}} \mathrm{h}^{2} \pm$ S.E. \\
\hline $\mathrm{A}$ & 1.290 & 8.010 & 9.300 & $0.14 \pm 0.03$ \\
$\mathrm{~B}$ & 0.003 & 0.029 & 0.032 & $0.10 \pm 0.04$ \\
K & 10.962 & 327.496 & 338.458 & $0.03 \pm 0.01$ \\
$\mathrm{IA}$ & 66.057 & 405.780 & 471.837 & $0.14 \pm 0.05$ \\
IW & 0.175 & 1.092 & 1.267 & $0.14 \pm 0.04$ \\
\hline
\end{tabular}

a A: Predicted mature live weight, B: Turning point of growth, K: Relative growth rate, IA: Inflection age, IW = Inflection weight.

${ }^{\mathrm{b}} \mathrm{h}^{2}$ : direct heritability; $\sigma_{a}^{2}$ : direct additive genetic variance; $\sigma_{e}^{2}$ : residual variance; $\sigma_{p}^{2}$ : phenotypic variance; S.E.: standard error.

\section{Genetic parameters}

\subsection{Univariate analyses}

Variance components and direct heritability estimates for the studied growth curve traits of Raeini Cashmere goat are presented in Table 2. The estimates of direct heritability were low, ranged from 0.03 for parameter K to 0.14 for parameter A, IA and IW. Direct heritability of parameter $\mathrm{B}$ was estimated as 0.10 . Among the parameters of the growth curve i.e. A, B and K, predicted mature live weight or A had the highest heritability than others. It is probably because of maturity at a later age in Raeini Cashmere goat, where the effect of environmental factors becomes relatively small. Low estimated values for direct heritability of the studied growth curve traits in Raeini Cashmere goat imply that direct selection for these traits may not be useful in terms of genetic change. Lupi et al. (2016) estimated direct heritability values of $0.41,0.51,0.62,0.41$ and 0.41 for A, B, P, IW and IA in the Segurena sheep breed, respectively, which were higher than the corresponding values estimated for Raeini Cashmere goat in the present study. Ghavi Hossein-Zadeh (2015) estimated medium direct heritability values of 0.39 and 0.23 for A and B parameters, and a low estimate of 0.034 for $\mathrm{K}$ parameter of growth curve in Iranian Guilan sheep. The estimates of direct heritability for A and B parameters of growth curve in Guilan sheep were higher than the corresponding estimates obtained in the present study for Raeini Cashmere goat but that of estimated for $\mathrm{K}$ parameter in Guilan sheep was in agreement with the obtained direct heritability for K parameter in Raeini Cashmere goat.

\subsection{Bivariate analyses}

The estimates of genetic and phenotypic correlations between the studied growth curve traits of Raeini Cashmere goat were presented in Table 3. Genetic correlations ranged from -0.76 (K-IW) to 0.98 (A-IW). In the present study, positive and high values were estimated for phenotypic (0.69) and genetic (0.73) correlations between A and B parameters of growth curve in Raeini Cashmere goats. Ghavi Hossein-Zadeh (2015) reported values of 0.57 and 0.47 for genetic and phenotypic correlations between A and B parameters of growth curve in Guilan sheep and pointed out that common genetic and physiological mechanisms control these traits. Positive genetic correlation between A and B parameter of growth curve in Raeini Cashmere goat implied that heavy kids at birth had greater mature weight. In other words, because of the positive and high genetic correlation between A and B, any increase in initial body weight of kids could be associated with increase in mature live weight.

Genetic and phenotypic correlations between parameters of $\mathrm{A}$ and $\mathrm{K}$ were estimated as -0.36 and -0.15 , respectively, implying that kids with faster growth rate were less likely to achieve as large a mature weight as kids that grew more slowly in early life. This finding is in line with Fitzhugh and Tylor (1971). Lupi et al. (2016) estimated values of -0.82 and -0.78 for genetic and phenotypic correlations between A and $\mathrm{K}$ parameters of growth curve (calculated via a logistic model) in Segureno sheep breed which were higher in magnitude than the corresponding estimated values in the present study. From a biological point of view, the correlation between parameters $\mathrm{A}$ and $\mathrm{K}$ of the growth curve is of crucial important. This correlation estimate implied that animals with higher mature body weights frequently represent lower body weight modification in relation to adult weight than animals with a lower mature weight (da Silva et al., 2012).

In the present study, phenotypic and genetic correlations for A-IA were not estimable due to not achieving convergence. Genetic and phenotypic correlation estimates of parameter A with IW were 0.98 and 0.68 , respectively implying high and positive relationship between mature weight and weight at inflection point of Raeini Cashmere goat and thus selection for weight at inflection point would improve mature weight of kids.

Genetic and phenotypic correlations for B-K were positive and low values of 0.11 and 0.13 , respectively. The estimates of genetic correlations of parameter B with IA and IW were 0.73 and 0.56 , respectively. The corresponding estimates of phenotypic correlations were 0.69 (BIA) and 0.42 (B-IW). Genetic correlation estimates of $\mathrm{K}$ with IA and with IW were -0.36 and -0.76 , respectively. The corresponding phenotypic correlations were also negative values of -0.15 and -0.30 , respectively. IA and IW had positive phenotypic $(0.86)$ and high genetic (0.99) correlations, implying IA and IW were highly correlated in terms of phenotypic and genetic effects.

\section{Conclusion}

Gompertz model showed a suitable fit to the body weight-age records in Raeini Cashmere goat. Although, Gompertz model adequately fit the growth curve in Raeini Cashmere goat, the predictions obtained in this study applying the growth curve parameters for the optimum age at slaughter and weight at maturity are different from real slaughter weight and weight at maturity. The studied growth curve parameters of Raeini Cashmere goat have shown low levels of additive genetic variation.

\section{Conflict of interest}

The authors declare that there are no conflicts of interests among authors and between authors and other people and organizations.

Table 3

Estimates of genetic (above diagonal) and phenotypic (below diagonal) correlations between the studied growth curve traits in Raeini Cashmere goat.

\begin{tabular}{|c|c|c|c|c|c|}
\hline Traits $^{\mathrm{a}}$ & A & B & $\mathrm{K}$ & IA & IW \\
\hline A & - & $0.73 \pm 0.20$ & $-0.36 \pm 0.06$ & NC & $0.98 \pm 0.17$ \\
\hline B & $0.69 \pm 0.02$ & - & $0.11 \pm 0.04$ & $0.73 \pm 0.20$ & $0.56 \pm 0.33$ \\
\hline $\mathrm{K}$ & $-0.15 \pm 0.03$ & $0.13 \pm 0.03$ & - & $-0.36 \pm 0.01$ & $-0.76 \pm 0.14$ \\
\hline IA & NC & $0.69 \pm 0.02$ & $-0.15 \pm 0.03$ & - & $0.99 \pm 0.17$ \\
\hline IW & $0.68 \pm 0.02$ & $0.42 \pm 0.03$ & $-0.30 \pm 0.03$ & $0.68 \pm 0.02$ & - \\
\hline
\end{tabular}

NC: Not Convergence.

${ }^{\text {a }}$ A: Predicted mature live weight, B: Turning point of growth, K: Relative growth rate, IA: Inflection age, IW = Inflection weight. 


\section{Acknowledgement}

The authors wish to thank all breeding Station staff of Raeini Cashmere goat which involved in data collection and maintaining the flock through the years.

\section{References}

Bertalanffy, L.V., 1957. Quantitative laws in metabolism and growth. Q. Rev. Biol. 32, 217-230.

Brody, S., 1945. Bioenergetics and Growth. Reinhold Publishing Corp, NY, USA.

Brown, J.E., Fitzhugh, Jr.H.A., Cartwright, T.C., 1976. A comparison of nonlinear models for describing weight-age relationships in cattle. J. Anim. Sci. 42, 810-818.

da Silva, L.S.A., Fraga, A.B., da Silva, F.D.L., Beelen, P.M.G., Silva, R.M.D.O., Tonhati, H., Barros, C.D.C., 2012. Growth curve in Santa Inês sheep. Small Rumin. Res. 105, 182-185.

Daskiran, I., Koncagul, S., Bingol, M., 2010. Growth characteristics of indigenous Norduz female and male lambs. J. Agric. Sci. 16, 62-69.

Fitzhugh, H.A., 1976. Analysis of growth curves and strategies for altering their shapes. J. Anim. Sci. 42, 1036-1051.

Fitzhugh, H.A., Taylor, S., 1971. Genetic analysis of degree of maturity. J. Anim. Sci. 33, $717-725$.

Gaddour, A., Ouni, M., Najari, S., 2012. Growth curve estimation in pure goat breeds and crosses at first and second generation under oases. J. Cell Anim. Biol. 6, 99-103.

Ghavi Hossein-Zadeh, N., 2015. Estimation of genetic relationships between growth curve parameters in Guilan sheep. J. Anim. Sci. Technol. 57, 19-24.

Gomez, D.A.A., Munoz, M.F.C., Betancur, L.F.R., 2008. Modelacion de funciones de crecimiento aplicadas a la produccion animal. Rev. Col. Cienc. Pec. 21, 39-58.

Kamjoo, B., Baneh, H., Yousefi, V., Mandal, A., Rahimi, G., 2014. Genetic parameter estimates for growth traits in Iran-black sheep. J. Appl. Anim. Res. 42, 79-88.

Karimkhani, I., Zahedifar, M., Lavaf, A., Karkoudi, K., 2005. A comparison between fattening performance of three goat breeds including Tali, Raeini and Black hair in Hormozgan province Karaj, Iran. Proceeding of the 2nd Conference of Sheep and Goat.

Keskin, I., Dag, B., Sariyel, V., Gokmen, M., 2010. Estimation of growth curve parameters in Konya Merino sheep. S. Afr. J. Anim. Sci. 39, 163-168.

Kume, K., Hajno, L., 2011. Study of growth curve variations for kids 0-6 months old of Alpine goat breed in Albania. Arch. Zootech. 13, 54-62.

Laird, A.K., 1965. Dynamics of relative growth. Growth 29, 249-263.

Lupi, T.M., Leon, J.M., Nogales, S., Barba, C., Delgado, J.V., 2016. Genetic parameters of traits associated with the growth curve in Segurena sheep. Animal 10, 729-735.

Meyer, K., 2007. WOMBAT - a tool for mixed model analyses in quantitative genetics by REML. J. Zhejiang Univ. Sci. B 8, 815-821.

Mignon-Grasteau, S.M., Piles, L., Varona, H., de Rochambeau, J.P., Poivey, Blasco, A., Beaumont, C., 2000. Genetic analysis of growth curve parameters for male and female chickens resulting from selection on shape of curve. J. Anim. Sci. 78, 2515-2524.

Najari, S., Gaddoun, A., Hamouda, M.B., Djemali, M., Khaldi, G., 2007. Growth model adjustment of local goat population under pastoral conditions in Tunisian arid zone. J. Agric. 6, 61-67.

Narinc, D., Karaman, E., Firat, M.Z., Aksoy, T., 2010. Comparison of non-linear growth models to describe the growth in Japanese quail. J. Anim. Vet. Adv. 9, 1961-1966.

Nelder, J.A., 1961. The fitting of a generalization of the logistic curve. Biometrics 17, 89-110.

Pires, L.C., Machado, T.M.M., Carneiro, P.L.S., da Silva, J.B.L., de Holanda, Barbosa, A.D., de Almeida Torres, R., 2017. Growth curve of Repartida goats reared in the Caatinga region. Braz. Semina: Ciencias Agrarias 38, 1041-1050.

Pittroff, W., Dahm, F., Blanc, F., Keisler, D., Cartwright, T.C., 2008. Onset of puberty and the inflection point of the growth curve in sheep-Brody's law revisited. J. Agric. Sci. 146, 239-250.

Raji, A.O., Asheikh, L.G., Mohammed, I.D., 2015. Comparison of growth of male and female kids of Nondescript goats in a semi-arid region of Nigeria. Iran. J. Appl. Anim. Sci. 5, 115-120.

Richards, F.J., 1959. A flexible growth function for empirical use. J. Exp. Bot. 10 290-300.

Sghaier, N., Gaddour, A., Mabrouk, O., Mouldi, A., Mohamed, B.H., 2007. Non genetic factors affecting local kid's growth curve under pastoral mode in Tunisian arid region. J. Biol. Sci. 7, 1005-1016.

Statistical Analysis System (SAS), 2004. SAS Users' Guide, Version 9.1. SAS Institute Inc. Cary, North Carolina, USA.

Waheed, A., Khan, M.S., Ali, S., Sarwar, M., 2011. Estimation of growth curve parameters in Beetal goats. Arch. Tierz. 54, 287-296. 